Research Article

\title{
Invariant Analysis, Analytical Solutions, and Conservation Laws for Two-Dimensional Time Fractional Fokker- Planck Equation
}

\author{
Nisrine Maarouf $(D)$ and Khalid Hilal $(i)$ \\ Laboratory of Applied Mathematics and Scientific Computing, Sultan Moulay Slimane University, P.O. Box 523, \\ 23000 Beni Mellal, Morocco
}

Correspondence should be addressed to Nisrine Maarouf; nisrine.maarouf6@gmail.com

Received 10 April 2021; Accepted 24 May 2021; Published 1 June 2021

Academic Editor: Andrea Scapellato

Copyright (c) 2021 Nisrine Maarouf and Khalid Hilal. This is an open access article distributed under the Creative Commons Attribution License, which permits unrestricted use, distribution, and reproduction in any medium, provided the original work is properly cited.

The main purpose of this paper is to apply the Lie symmetry analysis method for the two-dimensional time fractional FokkerPlanck (FP) equation in the sense of Riemann-Liouville fractional derivative. The Lie point symmetries are derived to obtain the similarity reductions and explicit solutions of the governing equation. By using the new conservation theorem, the new conserved vectors for the two-dimensional time fractional Fokker-Planck equation have been constructed with a detailed derivation. Finally, we obtain its explicit analytic solutions with the aid of the power series expansion method.

\section{Introduction}

Fractional calculus has attracted more attention of many researches in various scientific areas including biology, physics, financial theory, gas dynamics, engineering, fluid mechanics, and other areas of science, see for example [18]. The theory of fractional calculus is considered as a generalization of classical differential and integral calculus; it is an excellent tool for describing the memory effect and hereditary properties of various processes and viscoelastic materials. Due to its realistic senses, many researchers have tried to look for exact, analytical, and numerical solutions of fractional partial differential equations using different powerful methods such us $\left(G^{\prime} / G\right)$-expansion method [9], the Variational iteration method $[10,11]$, functional variable method [12], subequation method [13], Finite difference method [14], Exp function method [15], Homotopy analysis method [16], Adomian decomposition method [17], the First integral method [18], Laplace transform method [19], Sumudu transform method [20], and so many other approaches.
The Lie symmetry method was firstly advocated by the Norwegian mathematician Sophus Lie [21, 22], who has made great achievements in the theories of continuous groups and differential equations. It is an efficient approach and widely employed for solving ordinary differential equations (ODEs), partial differential equations (PDEs), and fractional partial differential equations (FPDEs). This popularity is due to its utility in determining the explicit solutions of both ODEs and PDEs, linearization of some nonlinear equations, reducing the order of independent variables, and so on. Many papers focused on constructing symmetries of different fractional differential equations [23, 24-27]. Furthermore, the concept of conservation laws is fundamental and widely used in the study of the resolution of PDEs. Moreover, they convey a large deal of information about the studied physical system. The new conservation laws were introduced by Ibragimov [28], based on the notion of Lie symmetry generators without Lagrangian for solving FPDEs. Therefore, this new conservation law plays an increasingly 
important role in solving the conservation laws of FPDEs. More details about conservation laws can be found in [29-32].

In this paper, we consider the following two-dimensional time fractional Fokker-Planck equation

$\frac{\partial^{\alpha} u}{\partial t^{\alpha}}=-\frac{a^{2} x^{2}}{2} \frac{\partial u^{2}}{\partial x^{2}}-\frac{b^{2} y^{2}}{2} \frac{\partial u^{2}}{\partial y^{2}}-k a b x y \frac{\partial u^{2}}{\partial x \partial y}-r x \frac{\partial u}{\partial x}-r y \frac{\partial u}{\partial y}+r u$

It is well known that the Markovian diffusion process can be described with the Fokker-Planck equation. The FokkerPlanck equation is a partial differential equation for the probability density and the transition probability of these stochastic processes. It plays an important role in control theory, fluid mechanics, astrophysics, and quantum [33]. Moreover, it has been applied in various natural science fields such as quantum optics, solid-state physics, chemical physics, theoretical biology, and circuit theory. It is firstly proposed by Fokker and Planck to characterize the Brownian motion of particles [34]. Many researchers have solved the FokkerPlanck equation using various powerful methods, for more details see [35-37].

The fractional derivatives described here are in the Riemann-Liouville sense of order $\alpha(\alpha>0)$, see [38, 39], which is defined by

$$
\begin{aligned}
& D^{\alpha} u(t, x)=\frac{\partial^{\alpha} u}{\partial t^{\alpha}}=\left\{\begin{array}{c}
\frac{\partial^{m} u}{\partial t^{m}}, \alpha=m \in \mathbb{N}, \\
\frac{1}{\Gamma(m-\alpha)} \frac{\partial^{m}}{\partial t^{m}}
\end{array}\right. \\
& \cdot \int_{0}^{t}(t-\tau)^{m-\alpha-1} u(\tau, x) d \tau, m-1<\alpha<m, m \in \mathbb{N}^{*}
\end{aligned}
$$

where $\Gamma(z)$ is the Gamma function defined by

$$
\Gamma(z)=\int_{0}^{+\infty} e^{-t} t^{z-1}
$$

The main motivation behind this article is to make use of the Lie symmetry method to get the infinitesimal generators, group invariant solutions for the time fractional twodimensional Fokker-Planck equation (FP), and to construct conservation laws given by Ibragimov [28]. Therefore, the new conserved vectors have been obtained using the new conservation theorem. Based on the power series method [32], the explicit power series solutions of the two-dimensional time fractional Fokker-Planck equation are derived.

The rest of this paper is organized as follows: in Section 2, we review some basic definitions of the Lie Symmetry method for fractional partial differential equations (FPDEs) and its properties. By employing the proposed method, Lie point symmetries of the Eq. (1) are obtained; by using similarity variables, the reduced equations are obtained; solving some of them, then the similarity solutions of Eq. (1) are deduced in Section 3. In Section 4, the conservation laws of
Eq. (1) are obtained. Section 5 is devoted to constructing the explicit analytical power series solutions. Some conclusions and discussions are given in Section 6.

\section{Method of Lie Symmetry Analysis for FPDEs}

In this section, we briefly review the main points about Lie symmetry analysis of FPDEs [39-41] of the following form

$$
D_{t}^{\alpha} u=F\left(x, y, t, u, u_{t}, u_{x}, u_{y}, u_{x x}, u_{y y}, \cdots\right), \alpha>0
$$

We assume that the Eq. (4) is invariant under a oneparameter $\varepsilon$ Lie group of infinitesimal transformations which are given as

$$
\begin{gathered}
\widehat{x}=x+\varepsilon \xi(x, y, t, u)+O\left(\varepsilon^{2}\right), \\
\hat{y}=y+\varepsilon \zeta(x, y, t, u)+O\left(\varepsilon^{2}\right), \\
\widehat{t}=t+\varepsilon \tau(x, y, t, u)+O\left(\varepsilon^{2}\right), \\
\widehat{u}=u+\varepsilon \eta(x, y, t, u)+O\left(\varepsilon^{2}\right), \\
\frac{\partial^{\alpha} \widehat{u}}{\partial t^{\alpha}}=\frac{\partial^{\alpha} u}{\partial t^{\alpha}}+\varepsilon \eta_{\alpha}^{0}(x, y, t, u)+O\left(\varepsilon^{2}\right), \\
\frac{\partial \widehat{u}}{\partial \widehat{x}}=\frac{\partial u}{\partial x}+\varepsilon \eta^{x}(x, y, t, u)+O\left(\varepsilon^{2}\right), \\
\frac{\partial \widehat{u}}{\partial \widehat{y}}=\frac{\partial u}{\partial y}+\varepsilon \eta^{y}(x, y, t, u)+O\left(\varepsilon^{2}\right), \\
\frac{\partial^{2} \widehat{u}}{\partial x \wedge^{2}}=\frac{\partial^{2} u}{\partial x^{2}}+\varepsilon \eta^{x x}(x, y, t, u)+O\left(\varepsilon^{2}\right), \\
\frac{\partial^{2} \widehat{u}}{\partial y \wedge^{2}}=\frac{\partial^{2} u}{\partial y^{2}}+\varepsilon \eta^{y y}(x, y, t, u)+O\left(\varepsilon^{2}\right),
\end{gathered}
$$

where $\varepsilon<<1$ is a group parameter and $\xi, \zeta$, $\eta$, and $\tau$ are infinitesimals and $\eta_{\alpha}^{0}$ is extended infinitesimal. The explicit expressions of $\eta^{x}, \eta^{y}, \eta^{x x}, \eta^{y y}$ are given by

$$
\begin{array}{cc}
\eta^{x} & =D_{x}(\eta)-u_{x} D_{x}(\xi)-u_{y} D_{x}(\zeta)-u_{t} D_{x}(\tau) \\
\eta^{y} & =D_{y}(\eta)-u_{x} D_{y}(\xi)-u_{y} D_{y}(\zeta)-u_{t} D_{y}(\tau) \\
\eta^{x x} & =D_{x}\left(\eta^{x x}\right)-u_{x x x} D_{x}(\xi)-u_{x x y} D_{x}(\zeta)-u_{x x t} D_{x}(\tau)
\end{array}
$$

$D_{x}, D_{y}$, and $D_{t}$ are the total derivatives with respect to $x, y$ , and $t$, respectively, which are defined as

$$
D_{x^{i}}=\frac{\partial}{\partial x^{i}}+u_{i} \frac{\partial}{\partial u}+u_{i j} \frac{\partial}{\partial u_{j}}+\cdots, i, j=1,2,3, \cdots,
$$

where $u_{i}=\partial u / \partial x^{i}, u_{i j}=\partial^{2} u / \partial x^{i} \partial x^{j}$, and so on. 
The infinitesimal generator $X$ is given by the following expression

$$
X=\xi(x, y, t, u) \frac{\partial}{\partial x}+\zeta(x, y, t, u) \frac{\partial}{\partial x}+\tau(x, y, t, u) \frac{\partial}{\partial t}+\eta(x, y, t, u) \frac{\partial}{\partial u} .
$$

The infinitesimal generator $X$ satisfy the following invariance condition of Eq. (4):

$$
\left.\operatorname{Pr}^{(n)} X(\Delta)\right|_{\Delta=0}=0, n=1,2, \cdots
$$

where

$$
\Delta:=D_{t}^{\alpha} u-F\left(x, t, u, u_{t}, u_{x}, u_{y}, u_{x x}, u_{y y} \cdots\right)
$$

The structure of the Riemann-Liouville derivative must be invariant under transformations (5), because the lower limit of the integral (2) is fixed. The invariance condition yields

$$
\left.\tau(x, y, t, u)\right|_{t=0}=0
$$

The explicit form of the $\alpha^{\text {th }}$ extended infinitesimal can be obtained as follows:

$$
\begin{aligned}
\eta_{\alpha}^{0}= & \frac{\partial^{\alpha} \eta}{\partial t^{\alpha}}+\left(\eta_{u}-\alpha D_{t}(\tau)\right) \frac{\partial^{\alpha} u}{\partial t^{\alpha}}-u \frac{\partial^{\alpha} \eta_{u}}{\partial t^{\alpha}} \\
& +\mu+\sum_{n=1}^{\infty}\left[\left(\begin{array}{c}
\alpha \\
n
\end{array}\right) \frac{\partial^{\alpha} \eta_{u}}{\partial t^{\alpha}}-\left(\begin{array}{c}
\alpha \\
n+1
\end{array}\right) D_{t}^{n+1}(\tau)\right] D_{t}^{\alpha-n}(u) \\
& -\sum_{n=1}^{\infty}\left(\begin{array}{c}
\alpha \\
n
\end{array}\right) D_{t}^{n}(\xi) D_{t}^{\alpha-n}\left(u_{x}\right)-\sum_{n=1}^{\infty}\left(\begin{array}{l}
\alpha \\
n
\end{array}\right) D_{t}^{n}(\zeta) D_{t}^{\alpha-n}\left(u_{y}\right),
\end{aligned}
$$

where

$$
\begin{aligned}
\mu= & \sum_{n=2}^{\infty} \sum_{m=2}^{n} \sum_{k=2}^{m} \sum_{r=0}^{k-1}\left(\begin{array}{l}
\alpha \\
n
\end{array}\right)\left(\begin{array}{l}
n \\
m
\end{array}\right)\left(\begin{array}{l}
k \\
r
\end{array}\right) \frac{1}{k !} \frac{t^{n-\alpha}}{\Gamma(n+1-\alpha)}(-u)^{r} \frac{\partial^{m}}{\partial t^{m}} \\
& \cdot\left(u^{k-r}\right) \frac{\partial^{n-m+k} \eta}{\partial x^{n-m} \partial u^{k}} .
\end{aligned}
$$

It is worth noting that $\mu=0$ if the infinitesimal $\eta$ is linear in $u$, due to the presence of $\partial^{k} \eta / \partial u^{k}$.

Definition 1 (see [42]). The function $u=\theta(\mathrm{x}, \mathrm{y}, \mathrm{t})$ is an invariant solution of Eq. (4) if and only if

(i) $u=\theta(x, y, t)$ is an invariant surface, that is to say

$$
\begin{aligned}
X \theta= & 0 \Longleftrightarrow\left(\xi(x, y, t, u) \frac{\partial}{\partial x}+\zeta(x, y, t, u) \frac{\partial}{\partial y}\right. \\
& \left.+\tau(x, y, t, u) \frac{\partial}{\partial t}+\eta(x, y, t, u) \frac{\partial}{\partial u}\right) \theta=0 .
\end{aligned}
$$

(ii) $u=\theta(x, y, t)$ satisfies Eq. (4)

\section{Symmetry Analysis and Similarity Reductions of the Two-Dimensional Time Fractional Fokker-Planck Equation}

In the present section, the Lie symmetry analysis method has been applied for deriving the infinitesimal generators of the two-dimensional time fractional Fokker-Planck (1). By using the third prolongation $[43,44]$, the symmetry determining equation for Eq. (1) has been obtained as

$$
\begin{aligned}
\eta_{0}^{\alpha}+ & \frac{a^{2} x^{2}}{2} \eta^{x x}+a^{2} x \xi u_{x x}+\frac{b^{2}}{2} y^{2} \eta^{y y}+b^{2} \varphi y u_{y y}+k a b x y \eta^{x y} \\
& +k a b(x \varphi+y \xi) u_{x y}+r x \eta^{x}+r \xi u_{x}+r y \eta^{y}+r y u_{y}-r u=0 .
\end{aligned}
$$

By substituting the expressions $\eta_{\alpha}^{0}$ given in Eq. (6) and Eq. (12) into Eq. (15), and equating various powers of derivatives of $u$ to zero, we obtain an overdetermined system of linear equations; by solving this system, we obtain the following infinitesimals

$$
\begin{array}{ll}
\eta(x, y, u, t)=\left(C_{1} \ln (x)+C_{2} \ln (y)+C_{3}\right) u+f(x, y, t), \\
\tau(x, y, u, t) & =-\frac{a b k\left(C_{1}+C_{3}\right)}{\alpha r} t+C_{4}, \\
\xi(x, y, u, t) & =\left(-\frac{C_{1} a b k \ln (x)}{r}+C_{6}\right) x, \\
\varphi(x, y, u, t) & =\left(-\frac{C_{3} a b k \ln (y)}{r}+C_{5}\right) y,
\end{array}
$$

where $C_{i}, i=1, \ldots, 6$ are arbitrary constants. So, the associated vector fields of Eq. (1) are given by

$$
\begin{aligned}
& X_{1}=y \frac{\partial}{\partial y} \\
& X_{2}=x \frac{\partial}{\partial x} \\
& X_{3}=\ln (x) \frac{\partial}{\partial u}+\frac{4 a^{2} t}{\alpha\left(a^{2}-2 r\right)} \frac{\partial}{\partial t}+\frac{2 a^{2} \ln (x)}{a^{2}-2 r} x \frac{\partial}{\partial x} \\
& X_{\infty}=f(x, y, t) \frac{\partial}{\partial u}
\end{aligned}
$$


Case 2. For $X_{1}=\partial / \partial y$, the characteristic equation is

$$
\frac{d y}{y}=\frac{d x}{0}=\frac{d u}{0}=\frac{d t}{0} .
$$

By solving the above characteristic equation, we obtain the solution $u=f(x, t)$. Substituting it into Eq. (1), we derive the following reduced fractional ordinary Fokker-Planck equation:

$$
D_{t}^{\alpha} f(x, t)=-\frac{b^{2} x^{2}}{2} \frac{\partial^{2} f}{\partial x^{2}}(x, t)-r x \frac{\partial f}{\partial x}(x, t)+r f(x, t)
$$

Using the symmetry method, we obtain the following infinitesimals:

$$
\begin{gathered}
\eta_{1}=\ln (x) M_{2} u+M_{1} u+f(x, t), \\
\tau_{1}=\frac{4 M_{2} a^{2} t}{\alpha\left(a^{2}-2 r\right)}+M_{3}, \\
\xi_{1}=\left(\frac{4 M_{2} a^{2} t}{\alpha\left(a^{2}-2 r\right)}+M_{4}\right) x,
\end{gathered}
$$

where $M_{i}, i=1, \ldots, 4$ are arbitrary constants. Then, the Lie algebra of infinitesimal symmetries of Eq. (19) is given by

$$
\begin{aligned}
& X_{11}=x \frac{\partial}{\partial x}, \\
& X_{12}=u \frac{\partial}{\partial u}, \\
& X_{13}=\ln (x) \frac{\partial}{\partial u}+\frac{2 a^{2} \ln (x)}{a^{2}-2 r} x \frac{\partial}{\partial x}+\frac{4 a^{2} t}{\alpha\left(a^{2}-2 r\right)} t \frac{\partial}{\partial t}, \\
& X_{\infty}=f(x, t) \frac{\partial}{\partial u} .
\end{aligned}
$$

Case 3. The characteristic equation for the infinitesimal generator $X_{11}$ can be expressed symbolically as follows:

$$
\frac{d x}{1}=\frac{d t}{0}=\frac{d u}{0}
$$

By solving the above characteristic equation, we obtain the solution $u=f(t)$. Substituting it into Eq. (19), we derive the reduced fractional ordinary equation:

$$
D_{t}^{\alpha} f=r f
$$

The above can be solved through the Laplace transform method

$$
\mathscr{L}\left(D_{t}^{\alpha} f\right)=r \mathscr{L}(f)
$$

Since the Laplace transform of the Riemann-Liouville deriv- ative is defined by the following form

$$
\mathscr{L}\left\{D_{x}^{\alpha} f(x), s\right\}=s^{\alpha} F(s)-\sum_{k=0}^{n-1} s^{k} D^{\alpha-k-1} f\left(0_{+}\right),
$$

then,

$$
\mathscr{L}\left(D_{t}^{\alpha} f\right)=s^{\alpha} \mathscr{L}(f)-s^{\alpha-1}
$$

According to Eq. (25), we have

$$
\mathscr{L}(f)=\frac{s^{\alpha-1}}{s^{\alpha}-r} .
$$

By using the inverse Laplace transform, it gives

$$
f(t)=E_{\alpha, 1}\left(r t^{\alpha}\right)
$$

where

$$
E_{\alpha, \beta}(z)=\sum_{k=0}^{\infty} \frac{z^{k}}{\Gamma(\alpha k+\beta)}, \alpha, \beta \in \mathbb{C},
$$

is the Mittag-Leffler function.

Case 4. For $X_{11}+\gamma X_{12}$, the similarity transformation corresponding to this generator can be derived by solving the associated characteristic equation

$$
\frac{d x}{x}=\frac{d u}{u}=\frac{d t}{t}
$$

which take the form

$$
u=x^{\gamma} g(t)
$$

replacing it in Eq. (19) yields the following reduced FODE:

$$
D_{t}^{\alpha}(g(t))=\left(-\frac{b^{2}}{2} \alpha(\alpha-1)-r(\alpha-1)\right) g(t)
$$

By using the Laplace transform, we obtain the following solution

$$
g(t)=E_{\alpha, 1}\left(\left(\frac{b^{2}}{2} \alpha(\alpha-1)+r(\alpha-1)\right) t^{\alpha}\right)
$$

\section{Conservation Laws of the Two-Dimensional Time Fractional Fokker-Planck Equation}

In this section, the conservation laws of the two-dimensional time fractional Fokker-Planck equation have been investigated by using a new conservation theorem [28]. The conserved vectors $C_{t}, C_{x}, C_{y}$ have been obtained, and it satisfies 
the following conservation equation:

$$
D_{t}\left(C^{t}\right)+D_{x}\left(C^{x}\right)+D_{y}\left(C^{y}\right)=0
$$

The formal Lagrangian for Eq. (1) can be written as follows:

$$
\begin{aligned}
L= & \omega(x, y, t)\left[\frac{\partial^{\alpha} u}{\partial t^{\alpha}}+\frac{a^{2} x^{2}}{2} \frac{\partial u^{2}}{\partial x^{2}}+\frac{b^{2} y^{2}}{2} \frac{\partial u^{2}}{\partial y^{2}}+k a b x y \frac{\partial u^{2}}{\partial x \partial y}\right. \\
& \left.+r x \frac{\partial u}{\partial x}+r y \frac{\partial u}{\partial y}-r u\right],
\end{aligned}
$$

where $\omega(x, y, t)$ is the new dependent variable. Based on the definition of the Lagrangian, the action integral of Eq. (35) is given by

$$
\int_{0}^{T} \int_{\Omega_{x}} \int_{\Omega_{y}} L\left(x, y, t, \omega, u, D_{t}^{\alpha} u, u_{x}, u_{y}, u_{x x}, u_{x y}, u_{y y}\right) d x d y d t
$$

The Euler-Lagrange operator is defined as

$$
\begin{gathered}
\frac{\delta}{\delta u}=\frac{\partial}{\partial u}+\left(D_{t}^{\alpha} u\right)^{*} \frac{\partial}{\partial D_{t}^{\alpha}}-D_{x} \frac{\partial}{\partial u_{x}}-D_{y} \frac{\partial}{\partial u_{y}} \\
+D_{x}^{2} \frac{\partial}{\partial u_{x x}}+D_{y}^{2} \frac{\partial}{\partial u_{y y}}+D_{x} D_{y} \frac{\partial}{\partial u_{x y}} .
\end{gathered}
$$

The adjoint operator $\left(D_{t}^{\alpha}\right)^{*}$ of is defined by

$$
\left(D_{t}^{\alpha}\right)^{*}=(-1)^{n} R_{T}^{n-\alpha}\left(D_{t}^{n}\right)={ }_{t}^{C} D_{T}^{\alpha}
$$

where $R_{T}^{n-\alpha}$ is the right-sided operator of fractional integration of order $(n-\alpha)$ that is defined by

$$
R_{T}^{n-\alpha} f(x, t)=\frac{1}{\Gamma(n-\alpha)} \int_{t}^{T}(\tau-t)^{n-\alpha-1} f(x, \tau) d \tau
$$

So, the adjoint equation of Eq. (1) as the Euler-Lagrange equation, given by

$$
\frac{\delta L}{\delta u}=0
$$

For the case of three independent variables $x, y, t$ and one dependent variable $u(x, y, t)$, we get

$$
\bar{X}+D_{t}(\tau) I+D_{x}(\xi) I+D_{y}(\zeta) I=W \frac{\delta}{\delta u}+D_{t}\left(C^{t}\right) I+D_{x}\left(C^{x}\right) I+D_{y}\left(C^{y}\right) I,
$$

where $I$ is the identity operator and $\delta / \delta u$ is denoted as the
Euler-Lagrange operator. So $\bar{X}$ is presented as

$$
\begin{aligned}
\bar{X}= & \xi \frac{\partial}{\partial x}+\tau \frac{\partial}{\partial t}+\eta \frac{\partial}{\partial u}+\zeta \frac{\partial}{\partial y}+\eta_{\alpha}^{0} \frac{\partial}{\partial D_{t}^{\alpha} u}+\eta^{x} \frac{\partial}{\partial u_{x}} \\
& +\eta^{x x} \frac{\partial}{\partial u_{x x}}+\eta^{y} \frac{\partial}{\partial u_{y}}+\eta^{y y} \frac{\partial}{\partial u_{y y}}+\eta^{x y} \frac{\partial}{\partial u_{x y}}
\end{aligned}
$$

and the Lie characteristic function $W$ is given by

$$
W=\eta-\tau u_{t}-\xi u_{x}-\zeta u_{y}
$$

Using the Lie symmetries $V_{1}, V_{2}, V_{3}$, we have

$$
\begin{aligned}
W_{1} & =-x u_{x}, W_{2}=-u_{t}, W_{3}=-y u_{y}, W_{4} \\
& =u \ln (x) \frac{\partial}{\partial u}+\frac{a b k}{\alpha r} t \frac{\partial}{\partial t}+\frac{a b k}{r} x \ln (x) \frac{\partial}{\partial x}, \\
W_{5} & =u \ln (y) \frac{\partial}{\partial u}+\frac{a b k}{\alpha r} t \frac{\partial}{\partial t}+\frac{a b k}{r} y \ln (y) \frac{\partial}{\partial y}, W_{6} \\
& =u, W_{\infty}=f(x, y, t) .
\end{aligned}
$$

Based on the fractional generalizations of the Noether operators, the components of conserved vectors can be presented as follows:

$$
\begin{aligned}
C^{t}= & \tau L+\sum_{k=0}^{n-1}(-1)^{k}{ }_{0} D_{t}^{\alpha-1-k}(W) D_{t}^{k} \frac{\partial L}{\partial_{0} D_{t}^{\alpha} u} \\
& -(-1)^{n} J\left(W, D_{t}^{k} \frac{\partial L}{\partial_{0} D_{t}^{\alpha} u}\right),
\end{aligned}
$$

where $J($.$) is defined by$

$$
J(f, g)=\frac{1}{\Gamma(n-\alpha)} \int_{0}^{t} \int_{t}^{T} \frac{f(\tau, x, y) g(\theta, x, y)}{(\theta-\tau)^{\alpha+1-n}} d \theta d \tau
$$

And the other components $C^{i}$ are defined as

$$
\begin{aligned}
C^{i}= & \xi^{i} L+W_{\theta}\left[\frac{\partial L}{\partial u_{i}^{\theta}}-D_{j}\left(\frac{\partial L}{\partial u_{i j}^{\theta}}\right)+D_{j} D_{k}\left(\frac{\partial L}{\partial u_{i j k}^{\theta}}\right)-\cdots\right] \\
& +D_{j}\left(W_{\theta}\right)\left[\frac{\partial L}{\partial u_{i j}^{\theta}}-D_{k}\left(\frac{\partial L}{\partial u_{i j k}^{\theta}}\right)+\cdots\right]+D_{j} D_{k}\left(W_{\theta}\right)\left(\frac{\partial L}{\partial u_{i j k}^{\theta}}-\cdots\right)+\cdots
\end{aligned}
$$

where $\xi^{1}=\xi, \xi^{2}=\zeta, \theta=1,2$. Using Eqs. (45) and (47), we obtain the following components of conserved vectors. 
Case 5. For $W_{1}=-x u_{x}$, we have

$$
\begin{aligned}
C^{t} & =\tau L{ }_{0} D_{t}^{\alpha-1}\left(W_{2}\right) D_{t}^{k} \frac{\partial L}{\partial_{0} D_{t}^{\alpha} u}+J\left(W_{2}, D_{t} \frac{\partial L}{\partial_{0} D_{t}^{\alpha} u}\right) \\
& =w(x, y, t) D_{t}^{\alpha-1}\left(-u_{t}\right)+J\left(-u_{t}, w_{t}\right), \\
C^{x}= & \xi L+W_{2}\left[\frac{\partial L}{\partial u_{x}}-D_{x} \frac{\partial L}{\partial u_{x x}}-D_{y} \frac{\partial L}{\partial u_{x y}}\right]+D_{x}\left(W_{2}\right)\left(\frac{\partial L}{\partial u_{x x}}\right) \\
& +D_{y}\left(W_{2}\right)\left(\frac{\partial L}{\partial u_{x y}}\right)=-u_{t}\left(r x w-\frac{a^{2} x^{2}}{2} w_{x}-a^{2} x w\right. \\
& \left.-k a b x y w_{y}-k a b x w\right)-u_{x t} w \frac{a^{2} x^{2}}{2}-u_{y t} k a b x y w \\
C^{y}= & \xi L+W_{2}\left[\frac{\partial L}{\partial u_{y}}-D_{x} \frac{\partial L}{\partial u_{x y}}-D_{y} \frac{\partial L}{\partial u_{y y}}\right]+D_{x}\left(W_{2}\right)\left[\frac{\partial L}{\partial u_{x y}}\right] \\
& +D_{y}\left(W_{2}\right)\left(\frac{\partial L}{\partial u_{y y}}\right)=-u_{t}\left[r y w-k a b x y w_{x}-k a b y w\right. \\
& \left.-\frac{b^{2} y^{2}}{2} w_{y}-b^{2} y w\right]-u_{x t} k a b x y w-u_{y t} \frac{b^{2} y^{2}}{2} w .
\end{aligned}
$$

Case 7. For $W_{2}=-y u^{y}$, we have

$$
\begin{aligned}
C^{t} & =\tau L+{ }_{0} D_{t}^{\alpha-1}\left(W_{3}\right) D_{t}^{k} \frac{\partial L}{\partial_{0} D_{t}^{\alpha} u}+J\left(W_{3}, D_{t} \frac{\partial L}{\partial_{0} D_{t}^{\alpha} u}\right) \\
= & -y w(x, y, t) D_{t}^{\alpha-1}\left(u_{y}\right)-J\left(y u_{y}, w_{t}\right), \\
C^{x}= & \xi L+W_{3}\left[\frac{\partial L}{\partial u_{x}}-D_{x} \frac{\partial L}{\partial u_{x x}}-D_{x} \frac{\partial L}{\partial u_{x x}}-D_{y} \frac{\partial L}{\partial u_{x y}}\right] \\
& +D_{x}\left(W_{3}\right)\left(\frac{\partial L}{\partial u_{x x}}\right)+D_{y}\left(W_{3}\right)\left(\frac{\partial L}{\partial u_{x y}}\right) \\
= & -u_{y}\left(r x y w-\frac{a^{2} x^{2}}{2} y w_{x}-a^{2} x y w-k a b x y^{2} w_{y}-k a b x y w\right. \\
& -u_{x y} y w \frac{a^{2} x^{2}}{2}-u_{y y} k a b x y^{2} w, \\
C^{y}= & \xi L+W_{3}\left[\frac{\partial L}{\partial u_{y}}-D_{x} \frac{\partial L}{\partial u_{x y}}-D_{y} \frac{\partial L}{\partial u_{y y}}\right]+D_{x}\left(W_{3}\right)\left[\frac{\partial L}{\partial u_{x y}}\right] \\
& +D_{y}\left(W_{3}\right)\left(\frac{\partial L}{\partial u_{y y}}\right)=-u_{y}\left[r y^{2} w-k a b x y^{2} w-k a b x y^{2} w_{x}\right. \\
& b^{2} y^{3} \\
2 & w_{y}-b^{2} y^{2} w \\
& -u_{x t} k a b y^{2} w-u_{y y} \frac{b^{2} y^{3}}{2} w .
\end{aligned}
$$

Case 8. For $W_{4}=u \ln (x)+(k a b / \alpha r) t u_{t}+(k a b / r) x \ln (x) u_{x}$, we have

$$
\begin{aligned}
C^{t}= & \tau L+{ }_{0} D_{t}^{\alpha-1}\left(W_{4}\right) D_{t}^{k} \frac{\partial L}{\partial_{0} D_{t}^{\alpha} u}+J\left(W_{4}, D_{t} \frac{\partial L}{\partial_{0} D_{t}^{\alpha} u}\right) \\
= & w(x, y, t) D_{t}^{\alpha-1}\left(-u \ln (x)+\frac{k a b}{\alpha r} t u_{t}+\frac{k a b}{r} x \ln (x) u_{x}\right) \\
& -J\left(-u \ln (x)+\frac{k a b}{\alpha r} t u_{t}+\frac{k a b}{r} x \ln (x), w_{t}\right), \\
C^{x}= & \xi L+W_{4}\left[\frac{\partial L}{\partial u_{x}}-D_{x} \frac{\partial L}{\partial u_{x x}}-D_{x} \frac{\partial L}{\partial u_{x x}}-D_{y} \frac{\partial L}{\partial u_{x y}}\right] \\
+ & D_{x}\left(W_{4}\right)\left(\frac{\partial L}{\partial \mathrm{u}_{x x}}\right)+D_{y}\left(W_{4}\right)\left(\frac{\partial L}{\partial u_{x y}}\right)=(-u \ln (x) \\
& \left.+\frac{k a b}{\alpha r} t u_{t}+\frac{k a b}{r} x \ln (x)\right)\left(r x w-\frac{a^{2} x^{2}}{2} w_{x}\right. \\
& \left.-a^{2} x w-k a b x y w_{y}-k a b x w\right)+\left(\frac{u}{x}+(\ln (x)+1) \frac{k a b}{r} u_{x}\right. \\
+ & \left.\frac{k a b}{\alpha r} t u_{x t}+\frac{k a b}{r} x \ln (x) u_{x x}\right) \frac{a^{2} x^{2}}{2} w+\frac{k a^{2} b^{2}}{\alpha r} k^{2} x y t w u_{t y} \\
+ & \frac{k a^{2} b^{2}}{r} k x^{2} y \ln (x) u_{x y},
\end{aligned}
$$

$$
\begin{aligned}
C^{y}= & \xi L+W_{4}\left[\frac{\partial L}{\partial u_{y}}-D_{x} \frac{\partial L}{\partial u_{x y}}-D_{y} \frac{\partial L}{\partial u_{y y}}\right]+D_{x}\left(W_{4}\right)\left[\frac{\partial L}{\partial u_{x y}}\right] \\
& +D_{y}\left(W_{4}\right)\left(\frac{\partial L}{\partial u_{y y}}\right)=\left(u \ln (x)+\frac{k a b}{\alpha r} t u_{t}+\frac{k a b}{r} x \ln (x) u_{x}\right) \\
& +\left[r y w-k a b y w-k a b x y w_{x}-\frac{b^{2} y^{2}}{2} w_{y}-b^{2} y w\right] \\
& +\left(\frac{u}{x}+(\ln (x)+1) \frac{k a b}{r} u_{x}+\frac{k a b}{\alpha r} t u_{x t}+\frac{k a b}{r} x \ln (x) u_{x x}\right) k a b x y w \\
& +\frac{k a^{2} b^{3}}{2 \alpha r} k t y^{2} w u_{t y}+\frac{k a b^{3}}{2 r} k w x y^{2} \ln (x) u_{x y} .
\end{aligned}
$$

Case 9. For $W_{5}=u \ln (y)+(k a b / \alpha r) t u_{t}+(k a b / r) y \ln (y) u_{y}$, we have

$$
\begin{aligned}
C^{t}= & \tau L+{ }_{0} D_{t}^{\alpha-1}\left(W_{5}\right) D_{t}^{k} \frac{\partial L}{\partial_{0} D_{t}^{\alpha} u}+J\left(W_{5}, D_{t} \frac{\partial L}{\partial_{0} D_{t}^{\alpha} u}\right) \\
= & w(x, y, t) D_{t}^{\alpha-1}\left(u \ln (y)+\frac{k a b}{\alpha r} t u_{t}+\frac{k a b}{r} y \ln (y) u_{y}\right) \\
& -J\left(u \ln (y)+\frac{k a b}{\alpha r} t u_{t}+\frac{k a b}{r} y \ln (y) u_{y}, w_{t}\right)
\end{aligned}
$$




$$
\begin{aligned}
C^{x}= & \xi L+W_{4}\left[\frac{\partial L}{\partial u_{x}}-D_{x} \frac{\partial L}{\partial u_{x x}}-D_{x} \frac{\partial L}{\partial u_{x x}}-D_{y} \frac{\partial L}{\partial u_{x y}}\right] \\
& +D_{x}\left(W_{5}\right)\left(\frac{\partial L}{\partial u_{x x}}\right)+D_{y}\left(W_{5}\right)\left(\frac{\partial L}{\partial u_{x y}}\right) \\
= & \left(u \ln (y)+\frac{k a b}{\alpha r} t u_{t}+\frac{k a b}{r} y \ln (y) u_{y}\right)\left(r x w-\frac{a^{2} x^{2}}{2} w_{x}\right. \\
& \left.-a^{2} x w-k a b x y w_{y}-k a b x w\right)+\left(\frac{u}{y}+(\ln (y)+1) \frac{k a b}{r} u_{y}\right. \\
& \left.+\frac{k a b}{\alpha r} t u_{y t}+\frac{k a b}{r} y \ln (y) u_{y y}\right) k x y a b w+\frac{k a b}{\alpha r} t u_{x t} \\
& +\frac{k a^{3} b}{2 r} k x^{2} y \ln (y) w u_{x y}, \\
C^{y}= & \xi L+W_{5}\left[\frac{\partial L}{\partial u_{y}}-D_{x} \frac{\partial L}{\partial u_{x y}}-D_{y} \frac{\partial L}{\partial u_{y y}}\right]+D_{x}\left(W_{5}\right)\left[\frac{\partial L}{\partial u_{x y}}\right] \\
& +D_{y}\left(W_{5}\right)\left(\frac{\partial L}{\partial u_{y y}}\right)=\left(u \ln (y)+\frac{k a b}{\alpha r} t u_{t}+\frac{k a b}{r} y \ln (y) u_{y}\right) \\
& +\left[r y w-k a b y w-k a b x y w_{x}-\frac{b^{2} y^{2}}{2} w_{y}-b^{2} y w\right] \\
& +\left(\frac{u}{y}+(\ln (y)+1) \frac{k a b}{r} u_{y}+\frac{k a b}{\alpha r} t u_{y t}+\frac{k a b}{r} y \ln (y) u_{y y}\right) \frac{b^{2} y^{2}}{2} w \\
& +\frac{k^{2} a^{2} b^{2}}{\alpha r} k t x y w u_{x t} .
\end{aligned}
$$

Case 10. For $W_{6}=u$, we have

$$
\begin{aligned}
C^{t}= & \tau L+_{0} D_{t}^{\alpha-1}\left(W_{6}\right) D_{t}^{k} \frac{\partial L}{\partial_{0} D_{t}^{\alpha} u}+J\left(W_{6}, D_{t} \frac{\partial L}{\partial_{0} D_{t}^{\alpha} u}\right) \\
= & w(x, y, t) D_{t}^{\alpha-1}(u)-J\left(u, w_{t}\right), \\
C^{x}= & \xi L+W_{6}\left[\frac{\partial L}{\partial u_{x}}-D_{x} \frac{\partial L}{\partial u_{x x}}-D_{x} \frac{\partial L}{\partial u_{x x}}-D_{y} \frac{\partial L}{\partial u_{x y}}\right] \\
& +D_{x}\left(W_{6}\right)\left(\frac{\partial L}{\partial u_{x x}}\right)+D_{y}\left(W_{6}\right)\left(\frac{\partial L}{\partial u_{x y}}\right) \\
& =u\left(r x w-\frac{a^{2} x^{2}}{2} w_{x}-a^{2} x w-k a b x y w_{y}-k a b x w\right) \\
& +\frac{a^{2} x^{2}}{2} w u_{x}+k a b x y w u_{y}, \\
C^{y}=\xi & +W_{6}\left[\frac{\partial L}{\partial u_{y}}-D_{x} \frac{\partial L}{\partial u_{x y}}-D_{y} \frac{\partial L}{\partial u_{y y}}\right]+D_{x}\left(W_{6}\right)\left[\frac{\partial L}{\partial u_{x y}}\right] \\
+ & D_{y}\left(W_{6}\right)\left(\frac{\partial L}{\partial u_{y y}}\right)=u\left[r y w-k a b y w-k a b x y w_{x}\right. \\
& \left.-\frac{b^{2} y^{2}}{2} w_{y}-b^{2} y w\right]+\frac{b^{2} y^{2}}{\alpha r} w u_{y}+k a b x y w u_{x} .
\end{aligned}
$$

Case 11. For $W_{\infty}=f(x, y, t)$, we have

$$
\begin{aligned}
C_{\infty}^{t}= & \tau L+_{0} D_{t}^{\alpha-1}(f(x, y, t)) D_{t}^{k} \frac{\partial L}{\partial_{0} D_{t}^{\alpha} u}+J\left(f(x, y, t), D_{t} \frac{\partial L}{\partial_{0} D_{t}^{\alpha} u}\right) \\
= & w(x, y, t) D_{t}^{\alpha-1}(f(x, y, t))-J\left(f(x, y, t), w_{t}\right), \\
C_{\infty}^{x}= & \xi L+W_{\infty}\left[\frac{\partial L}{\partial u_{x}}-D_{x} \frac{\partial L}{\partial u_{x x}}-D_{x} \frac{\partial L}{\partial u_{x x}}-D_{y} \frac{\partial L}{\partial u_{x y}}\right] \\
& +D_{x}\left(W_{\infty}\right)\left(\frac{\partial L}{\partial u_{x x}}\right)+D_{y}\left(W_{\infty}\right)\left(\frac{\partial L}{\partial u_{x y}}\right) \\
= & f(x, y, t)\left(r x w-\frac{a^{2} x^{2}}{2} w_{x}-a^{2} x w-k a b x y w_{y}-k a b x w\right) \\
& +\frac{a^{2} x^{2}}{2} w f_{x}(x, y, t)+k a b x y w f_{y}(x, y, t), \\
C_{\infty}^{y}= & \xi L+W_{\infty}\left[\frac{\partial L}{\partial u_{y}}-D_{x} \frac{\partial L}{\partial u_{x y}}-D_{y} \frac{\partial L}{\partial u_{y y}}\right]+D_{x}\left(W_{\infty}\right)\left[\frac{\partial L}{\partial u_{x y}}\right] \\
& +D_{y}\left(W_{\infty}\right)\left(\frac{\partial L}{\partial u_{y y}}\right)=f(x, y, t)\left[r y w-k a b y w-k a b x y w_{x}\right. \\
& \left.-\frac{b^{2} y^{2}}{2} w_{y}-b^{2} y w\right]+\frac{b^{2} y^{2}}{\alpha r} w f_{y}(x, y, t)+f_{x}(x, y, t) k a b x y w .
\end{aligned}
$$

\section{Power Series and Analytical Solutions for Eq. (1)}

In this section, based on the power series method [45], the exact analytic solutions are a kind of exact power series solutions for Eq. (1), constructed with a detailed derivation.

$$
u(x, y, t)=u(w), w=m y+\rho x-\frac{\epsilon t^{\alpha}}{\Gamma(1+\alpha)},
$$

where $m, k, \alpha$, and $\epsilon \neq 0$ are arbitrary. The time fractional Fokker-Planck Eq. (1) is reduced to the following ODE

$$
(-\epsilon+r x \rho+r y m) u^{\prime}+\left(\frac{a^{2} x^{2}}{2} \rho^{2}+\frac{b y^{2}}{2} m^{2}+\rho a b k m x y\right) u^{\prime \prime}-r u=0 .
$$

We assume that the solution of Eq. (1) has the following form:

$$
u(w)=\sum_{n=0}^{\infty} \sigma_{n} \xi^{n}
$$

where $\sigma_{n}$ are constants to be determined later. According to 
Eq. (57), we get

$$
\begin{gathered}
u^{\prime}(w)=\sum_{n=0}^{\infty}(n+1) \sigma_{n+1} \xi^{n}, \\
u^{\prime \prime}(w)=\sum_{n=0}^{\infty}(n+1)(n+2) \sigma_{n+2} \xi^{n} .
\end{gathered}
$$

Substituting (57) and (58) into (56), we obtain

$$
\begin{aligned}
(-\epsilon+ & r x \rho+r y m) \sum_{n=0}^{\infty}(n+1) \sigma_{n+1} \xi^{n} \\
& +\left(\frac{a^{2} x^{2}}{2} \rho^{2}+\frac{b y^{2}}{2} m^{2}+\rho a b k m x y\right) \sum_{n=0}^{\infty}(n+1)(n+2) \sigma_{n+2} \xi^{n} \\
& -r \sum_{n=0}^{\infty} \sigma_{n} \xi^{n}=0
\end{aligned}
$$

Observing coefficients in Eq. (59), when $n=0$, we have

$$
(-\epsilon+r x \rho+r y m) \sigma_{1}+2\left(\frac{a^{2} x^{2}}{2} \rho^{2}+\frac{b y^{2}}{2} m^{2}+\rho a b k m x y\right) \sigma_{2}-r \sigma_{0}=0 .
$$

By comparing coefficients of $\sigma$, we get

$$
\sigma_{2}=\frac{(\epsilon-r x \rho-r y m) \sigma_{1}+r \sigma_{0}}{a x^{2} \rho^{2}+b m^{2} y^{2}+2 \rho a b k m x y} .
$$

When $n \geq 1$, we have

$$
\sigma_{n+2}=\frac{2}{(n+1)(n+2)} \frac{(\epsilon-r x \rho-r y m)(n+1) \sigma_{n+1}+r \sigma_{n}}{a x^{2} \rho^{2}+b m^{2} y^{2}+2 \rho a b k m x y}
$$

The power series solution for Eq. (5) can be rewritten as follows:

$$
\begin{aligned}
u(x, y, t)= & a_{0}+a_{1}\left(k x+m y-\frac{\epsilon t^{\alpha}}{\Gamma(1+\alpha)}\right) \\
& +\frac{(\epsilon-r x k-r y m) \sigma_{1}+r \sigma_{0}}{a x^{2} k^{2}+b y^{2} m^{2}+2 \rho x y a b k m}\left(k x+m y-\frac{\epsilon t^{\alpha}}{\Gamma(1+\alpha)}\right)^{2} \\
& +\sum_{n=1}^{\infty} \frac{1}{(n+1)(n+2)} \frac{2(\epsilon-r x k-r y m)(n+1) \sigma_{n+1}+r \sigma_{n}}{a x^{2} k^{2}+b y^{2} m^{2}+2 k a b x y k m} \\
& \cdot\left(k x+m y-\frac{\epsilon t^{\alpha}}{\Gamma(1+\alpha)}\right)^{n+2} .
\end{aligned}
$$

\section{Conclusion}

In this paper, the invariance properties of the twodimensional time fractional Fokker-Planck equation with the Riemann-Liouville fractional derivative have been investigated in the sense of Lie point symmetries. Then, the power series method has been applied to get an explicit solution for the two-dimensional time fractional Fokker-Planck equation. For obtaining new components of conserved vectors, a new theorem of conservation law has been employed along with the formal Lagrangian, which allows us to construct conservation laws for the two-dimensional time fractional FokkerPlanck equation. Our results show that the extended Lie group analysis approach and the power series method provide powerful mathematical tools to investigate other FDEs in different fields of applied mathematics. In addition, it shows that the proposed analysis is very efficient to construct conservation laws of the two-dimensional time fractional Fokker-Planck equation. Moreover, we can employ symmetry analysis to the time-space fractional Fokker-Planck equation; it will be valuable as future subject works.

\section{Data Availability}

No data were used to support this study.

\section{Conflicts of Interest}

The authors declare that they have no conflicts of interest.

\section{References}

[1] Y. Benia, M. Yassine, and A. Scapellato, "Existence of solution to Korteweg-de Vries equation in a non-parabolic domain," Nonlinear Analysis, vol. 195, article 111758, 2020.

[2] Y. Benia, M. Ruggieri, and A. Scapellato, "Exact solutions for a modified Schrödinger equation," Mathematics, vol. 7, no. 10, p. $908,2019$.

[3] M. Bouaouid, M. Hannabou, and K. Hilal, "Nonlocal conformable-fractional differential equations with a measure of noncompactness in Banach spaces," Journal of Mathematics, vol. 2020, Article ID 615080, 6 pages, 2020.

[4] R. Bagley and P. Torvik, "A theoretical basis for the application of fractional calculus to visco-elasticity," Journal of Rheology, vol. 27, no. 3, pp. 201-210, 1983.

[5] L. Debnath, "Recent applications of fractional calculus to science and engineering," International Journal of Mathematics and Mathematical Sciences, vol. 54, 3442 pages, 2003.

[6] M. Hannabou, K. Hilal, and A. Kajouni, "Existence results of hybrid fractional sequential integro-differntial equations," Engineering Mathematics Letters, vol. 2020, 2020.

[7] R. Hilfer, "Applications of fractional calculus in physics," World Scientific, Singapore, vol. 35, no. 12, pp. 87-130, 2000.

[8] A. Jhangeer, A. Hussain, M. Junaid-U-Rehman, D. Baleanu, and M. B. Riaz, "Quasi-periodic, chaotic and travelling wave structures of modified Gardner equation," Chaos, Solitons \& Fractals, vol. 143, p. 110578, 2021.

[9] N. Shang and B. Zheng, "Exact solutions for three fractional partial differential equations by the $\left(G^{\prime} / G\right)$ method," International Journal of Applied Mathematics, vol. 43, no. 3, pp. 114119, 2013.

[10] M. Hassouna, A. Ouhadan, and E. H. El Kinani, "On the solution of fractional order SIS epidemic model," Chaos, Solitons \& Fractals, vol. 117, pp. 168-174, 2018.

[11] C. C. Wu, "A fractional variational iteration method for solving fractional nonlinear differential equations," Computers \& Mathematics with Applications, vol. 61, no. 8, pp. 2186-2190, 2011. 
[12] W. Liu and K. Chen, "The functional variable method for finding exact solutions of some nonlinear time-fractional differential equations," Pramana, vol. 81, no. 3, pp. 377384, 2013.

[13] B. Tang, Y. He, L. Wei, and X. Zhang, "A generalized fractional sub-equation method for fractional differential equations with variable coefficients," Physics Letters A, vol. 376, no. 38-39, pp. 2588-2590, 2012.

[14] M. M. Meerschaert, H. P. Scheffler, and C. Tadjeran, "Finite difference methods for two-dimensional fractional dispersion equation," Journal of Computational Physics, vol. 211, no. 1, pp. 249-261, 2006.

[15] E. Aksoy, M. Kaplan, and A. Bekir, "Exponential rational function method for space-time fractional differential equations," Waves in Random and Complex Media, vol. 26, no. 2, pp. 142-151, 2016.

[16] S. Momani and Z. Odibat, "Homotopy perturbation method for nonlinear partial differential equations of fractional order," Physics Letters A, vol. 365, no. 5-6, pp. 345-350, 2007.

[17] S. S. Ray, "Analytical solution for the space fractional diffusion equation by two-step Adomian decomposition method," Communications in Nonlinear Science and Numerical Simulation, vol. 14, no. 4, pp. 1295-1306, 2009.

[18] M. Eslami, B. F. Vajargah, M. Mirzazadeh, and A. Biswas, "Application of first integral method to fractional partial differential equations," Indian Journal of Physics, vol. 88, no. 2, pp. 177-184, 2014.

[19] G. Jumarie, "Laplace's transform of fractional order via the Mittag-Leffler function and modified Riemann-Liouville derivative," Applied Mathematics Letters, vol. 22, no. 11, pp. 1659-1664, 2009.

[20] H. Bulut, H. M. Baskonus, and F. B. M. Belgacem, "The analytical solution of some fractional ordinary differential equations by the Sumudu transform method," Abstract and Applied Analysis, vol. 2013, Article ID 203875, 6 pages, 2013.

[21] G. W. Bluman and S. Kumei, Symmetries and Differential Equations, Springer, New York, NY, USA, 1989.

[22] P. J. Olver, Application of Lie Groups to Differential Equation, Springer, New York, NY, USA, 1986.

[23] J.-J. Mao, S.-F. Tian, T.-T. Zhang, and X.-J. Yan, "Lie symmetry analysis, conservation laws and analytical solutions for chiral nonlinear Schrödinger equation in $(2+1)$-dimensions," Nonlinear Analysis: Modelling and Control, vol. 25, no. 3, pp. 358-377, 2020.

[24] D. Baleanu, M. İnç, A. Yusuf, and A. I. Aliyu, "Time fractional third-order evolution equation: symmetry analysis, explicit solutions, and conservation laws," Journal of Computational and Nonlinear Dynamics, vol. 13, no. 2, 2018.

[25] M. I. El Bahi and K. Hilal, "Lie symmetry analysis, exact solutions, and conservation laws for the generalized timefractional KdV-like equation," Journal of Function Spaces, vol. 2021, Article ID 6628130, 10 pages, 2021.

[26] N. Maarouf, H. Maadan, and K. Hilal, "Lie symmetry analysis and explicit solutions for the time-fractional regularized longwave equation," International Journal of Differential Equations, vol. 2021, Article ID 6614231, 11 pages, 2021.

[27] S. Rashidi and S. R. Hejazi, "Analyzing Lie symmetry and constructing conservation laws for time-fractional Benny-Lin equation," International Journal of Geometric Methods in Modern Physics, vol. 14, no. 12, article 1750170, 2017.
[28] N. H. Ibragimov, "A new conservation theorem," Journal of Mathematical Analysis and Applications, vol. 333, no. 1, pp. 311-328, 2007.

[29] O. H. EL-Kalaawy, S. M. Moawad, M. M. Tharwat, and R. B. Al-Denari, "Conservation laws, analytical solutions and stability analysis for the time-fractional Schamel-Zakharov-Kuznetsov-Burgers equation," Advances in Difference Equations, vol. 2019, no. 1, 2019.

[30] B. Guo, H. Dong, and Y. Fang, "Symmetry groups, similarity reductions, and conservation laws of the time-fractional Fujimoto-Watanabe equation using lie symmetry analysis method," Complexity, vol. 2020, Article ID 4830684, 9 pages, 2020.

[31] W. Rui, P. Zhao, and Y. Zhang, "Invariant solutions and conservation laws of the $(2+1)$-dimensional Boussinesq equation," Abstract and Applied Analysis, vol. 2014, Article ID 840405, 6 pages, 2014.

[32] S. Sahoo and S. Saha Ray, "Invariant analysis with conservation law of time fractional coupled Ablowitz-Kaup-NewellSegur equations in water waves," Waves in Random and Complex Media, vol. 30, no. 3, pp. 530-543, 2020.

[33] C. Montagnon, "A closed solution to the Fokker-Planck equation applied to forecasting," Physica A: Statistical Mechanics and its Applications, vol. 420, pp. 14-22, 2015.

[34] A. P. Smirnov, A. B. Shmelev, and E. Ya Sheinin, "Analysis of Fokker-Planck approach for foreign exchange market statistics study," Physica A: Statistical Mechanics and its Applications, vol. 344, no. 1-2, pp. 203-206, 2004.

[35] P. Kumar and S. Narayanan, "Solution of Fokker-Planck equation by finite element and finite difference methods for nonlinear systems," Sadhana, vol. 31, no. 4, pp. 445-461, 2006.

[36] B. F. Spencer and L. A. Bergman, "On the numerical solution of the Fokker-Planck equation for nonlinear stochastic systems," Nonlinear Dynamics, vol. 4, no. 4, pp. 357-372, 1993.

[37] L. Yan, "Numerical solutions of fractional Fokker-Planck equations using iterative Laplace transform method," Abstract and Applied Analysis, vol. 2013, Article ID 465160, 7 pages, 2013.

[38] V. S. Kiryakova, Generalised Fractional calculus and Applications, CRC Press, 1993.

[39] K. S. Miller and B. Ross, An Introduction to the Fractional Calculus and Fractional Differential Equations, John Wiley and Sons, New York, NY, USA, 1993.

[40] K. Diethelm, The Analysis of Fractional Differential Equations, Springer, Berlin, Germany, 2010.

[41] I. Podlubny, Fractional Differential Equations, Academic Press, San Diego, CA, USA, 1999.

[42] G. W. Wang, "Lie symmetry analysis to the time fractional generalized fifth-order KdV equation," Communications in Nonlinear Science and Numerical Simulation, vol. 18, no. 9, pp. 2321-2326, 2013.

[43] R. K. Gazizov, A. A. Kasatkin, and S. Y. Lukashchuk, "Continuous transformations groups of fractional differential equations," Vestnik Usatu, vol. 9, pp. 125-135, 2007.

[44] R. K. Gazizov, A. A. Kasatkin, and S. Y. Lukashchuk, "Symmetry properties of fractional diffusion equations," Physica Scripta, vol. T136, article 014016, 2009.

[45] V. A. Galaktionov and S. R. Svirshchevskii, Exact Solutions and Invariant Subspaces of Nonlinear Partial Differential Equations in Mechanics and Physics, CRC Press, 2006. 